\title{
Vortex dynamics and elliptical structure wake interaction in the proximity of wall using 2-D RANS simulation
}

\author{
Arunkumar H. S. ${ }^{1^{*}}$, Chidanand Mangrulkar ${ }^{2}$ and Trushar Gohil ${ }^{2}$ \\ ${ }^{1}$ Deptarment of Mechanical Engineering, Manipal Institute of Technology, Manipal Academy of \\ Higher Education, Manipal, Udupi, Karnataka, India \\ ${ }^{2}$ Dept. of Mechanical Engg., Visvesvaraya National Institute of Technology, Nagpur, Maharashtra, \\ India
}

\begin{abstract}
The 2-D numerical study is performed to analyses the flow characteristic behind the elliptical structure placed near the wall for three different gap ratios as $0.25,0.5$, and 1.0. Computational domain and model is initially validated with the unbounded flow over a cylinder without considering wall effect for Reynolds number of 3900. For flow over the cylinder with near wall, computational domain is modelled as Blasius profile is the input to the area of interest. At different gap ratios the effect of boundary layer on vortex shedding is studied with Reynolds number of 1440. By applying different turbulent model for analysis, study the variation in the results and suggest the suitable model for the present type of study. It has been observed that the wall effect is predominant in case of the gap ratio of 0.25 as compared to other gap ratios.
\end{abstract}

Keywords: CFD, Elliptical structure, Cross flow, Wake

\section{Introduction}

Flow across the different type of cylindrical structure is a very common in most of the engineering application which comprises of off shore structures, pipelines, economizer, condenser coils, shell and tube heat exchangers, and Heating, Ventilation, Air Conditioning $\&$ Refrigeration (HVAC\&R) applications. So the laminar and turbulent viscous flow across these structure is a great interest with immense potential in research. As the flow is viscous, the hydrodynamics associated with the plate viscous layer and turbulence created at the cylinder downstream is studied experimentally and numerically by number of researchers. The flow in most of the application is comparatively low, and Reynolds number is usually less than 2000. Md. Mahbubar Rahman et al. [1], numerically investigated flow over a cylinder using 2-D finite volume basis, and estimated the pressure and drag coefficient. However research also continues in case of the rotating cylinder, as Atul Sharmal et al. [2] investigated numerically heat flow and fluid flow around a rotating cylinder for Reynolds number from 20-160 maintained at constant temperature, and concluded that rotation acts as drag reduction and heat transfer suppression. Gurunath Gandikota et al. [6] investigated

*Corresponding author: arunkumar.hs@manipal.edu 
vortex shedding phenomena around a cylinder subjected to heating/cooling and fixed in a vertical channel. Atul Sharma et al. [7] studied effect of channel-confinement of various degree for the case of upwind flow around a heated/cooled square cylinder for Reynolds number $=100$ and Prandle number $=0.7$.

In majority of the application, the cylinder is often supported of an additional domain wall in terms of adjacent cylinder or an external plate. Such a system is of lower importance and has not been explored much. S. Sarkar et al. [3], studied vortex dynamics of a wake formed by circular cylindrical object near to a plate boundary at Reynolds number equal to 1440 , for the different gap ratios (G/D) ( $\mathrm{G}$ is the gap between the wall and the cylindrical object, and $\mathrm{D}$ is the hydraulic diameter) by means of LES simulation. The use of the LES simulation is very costly and time consuming. Hence there is a need to perform the simpler and relatively cheaper analysis by means of the RANS simulation.

Literature study shows the different approach and method to analyse the flow. The aim of the present work is to numerically simulate the flow across the cylinder along with the domain boundary wall and results are analysed with the Large Eddy Simulation results and experimental available data. In the same fluid domain check the effect of different shape (Ellipse) objects effect on the boundary layer formation by means of 2-D RANS simulation. The domain wall is at a distance of $0.25 \mathrm{D}, 0.5 \mathrm{D}$, and $1.0 \mathrm{D}$ from the wall of the object.

\section{Methodology}

The flow field across a cylinder in two dimensional, with axis of the elliptical body perpendicular to the flow path. The fluid which passing over the elliptical body is air. It is treated to be incompressible and the flow is from left side of the computational domain. Computational domain is developed for unbounded flow over a cylinder by maintaining distance $8 \mathrm{D}$ in the inflow and $25 \mathrm{D}$ at the outflow and $8 \mathrm{D}$ between top and bottom of the wall. The flow over a circular cylinder is performed at a Reynolds number of 3900 with different turbulent models and compare the results with experimental results of Ma et al. (2000) [5].

Similarly computational domain is developed for elliptical structure with the wall at different relative gap distance $(\mathrm{G} / \mathrm{D}=0.25, \mathrm{G} / \mathrm{D}=0.5$ and $\mathrm{G} / \mathrm{D}=1.0)$. The figure (1) indicates the physical domain of the cylinder along with the wall boundary. Elliptical object dimensions are taken by maintaining same hydraulic diameter as the cylinder which is used for validation of the model with experiment results. Computational domain modelled as $10 \mathrm{D}$ in the upstream and $25 \mathrm{D}$ at the downstream of the cylinder in the direction of the flowing fluid. The height of the domain is $8.5 \mathrm{D}$ for gap ratio $(\mathrm{G} / \mathrm{D})$ of 0.25 , and 0.5 . Computational domain is extended to $9.0 \mathrm{D}$ for relative gap $(\mathrm{G} / \mathrm{D})$ of 1.0 . The above stated computational space is discretized using finite volume method. 


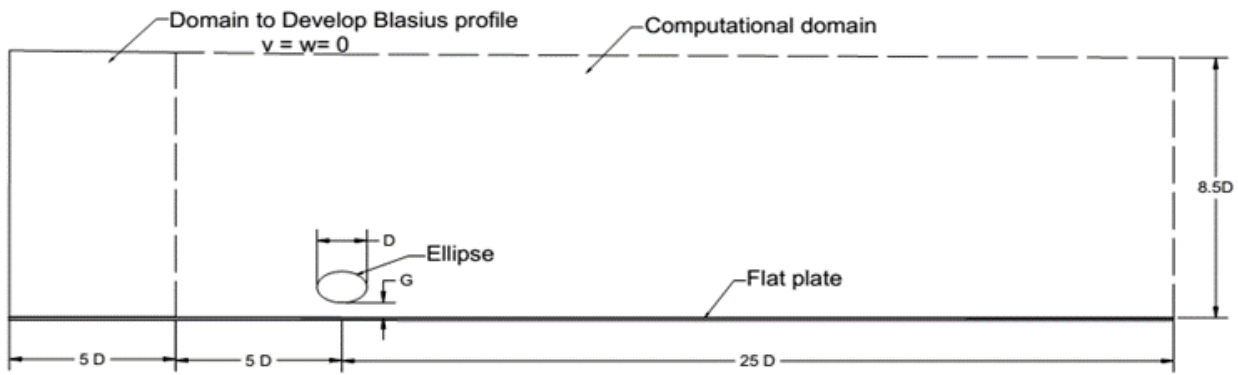

Fig. 1. Schematic diagram of the computational domain

The body fitted Cartesian uniform mesh is generated using partition block method in ICEM CFD ANSYS 16.0. The mesh is much finer nearer to the elliptical structure wall and plate boundary. The grid is formed by regulating the mesh size and structure and quality of mesh is obtained 0.9. Grid independent study is also carried out and typical mesh structure near the elliptical structure and plate as shown in figure 2.

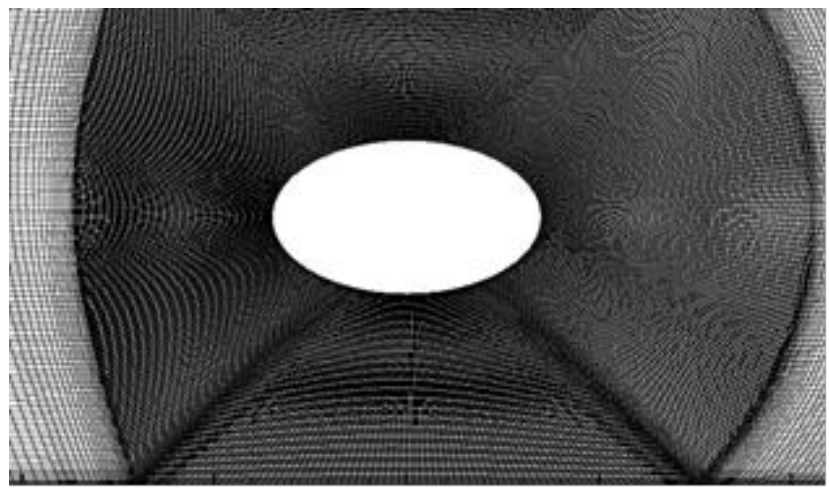

Fig. 2. Schematic diagram of the mesh structure around the Elliptical structure

\subsection{Governing equation and boundary condition}

The 2-D Navier-Stoke governing equation was discretized and further processed in ANSYS FLUENT 16.0. Very fine mesh around the elliptical object and near to the plat boundaries give better and accurate results. For present study enhanced wall treatment condition is applied by considering viscous boundary layer $y+$ as 1 . The solution accuracy of the CFD simulation is improved significantly by maintaining the $y^{+}=1$. The governing equations for momentum is discretized with the second order upwind scheme. The Pressure-velocity coupled algorithm is applied to get the combined effect of pressure and velocity components. In this numerical study, solution residuals are converged of the order of 10-6.

The Reynolds number is maintained at 1440, which depends on the free stream velocity of the working fluid i.e., air. The Blasius profile $(\mathrm{v}=\mathrm{w}=0)$ is applied by extending existing computation domain to $5 \mathrm{D}$ in upstream direction. The bottom surface of the computation space is treated with no-slip boundary condition, while upper is specified shear. 


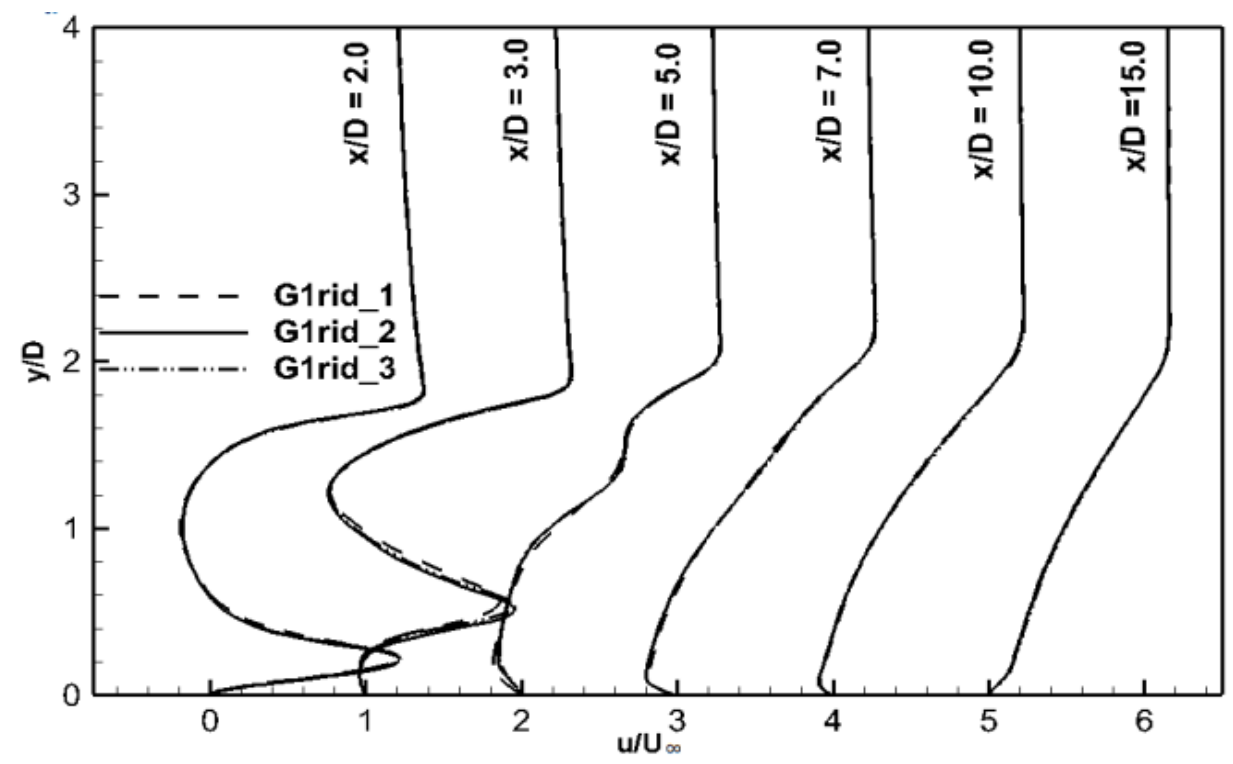

Fig. 3. Grid-independent test for basic model for relative gap ratio $=0.25$ and Reynolds number $=$ 1440. (Grid_1=40000 elements; Grid_2=75000 elements; Grid_3 = 95000 elements.)

Figure 3 indicates the grid independence performed by considering three different mesh size, as 40000, 75000, and 95000 number of elements. It has been observed from the graph that the deviation in velocity ratio is nominal as the number of elements increased to 95000 from 75000 . Hence the size of the grid comprising of 75000 number of elements is selected for the numerical simulation.

\section{Results and Discussion}

The numerical analysis is initially performed for the unbounded cylinder by considering the various two equation turbulence model such as RNG k-epsilon, Std. k-epsilon, Realizable k-epsilon, and k-omega models at Reynolds number of 3900.
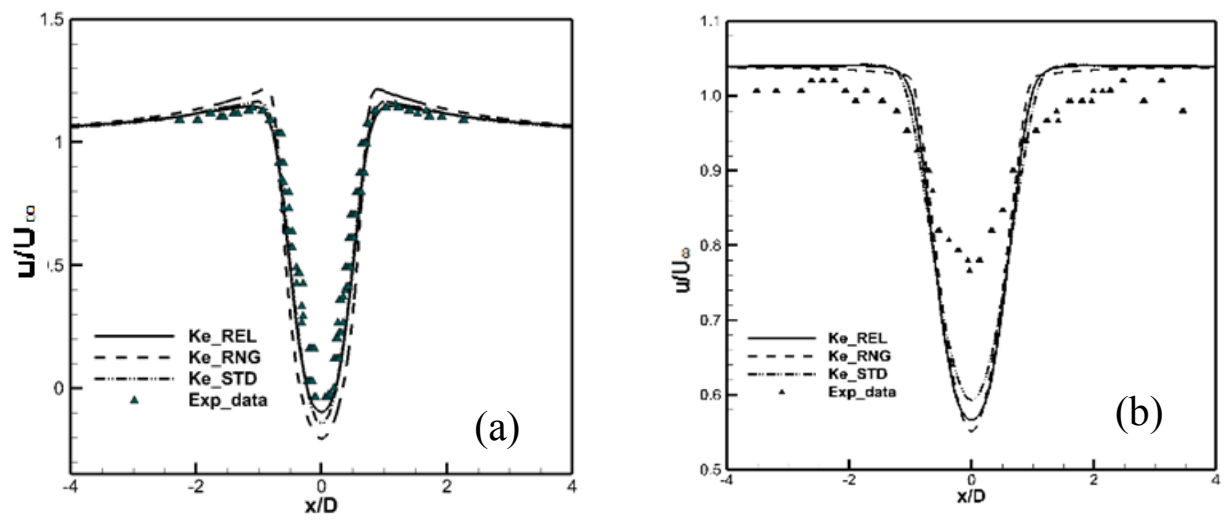

Fig. 4. Free stream velocity profile at (a) $y / D=1.06$ (b) $y / D=7.0$ 
From figure 4, it is clear that the plot for free stream velocity k-E Realizable model is in close proximity compared to the experimental result obtained to Ma et al. (2000) [5]. Realizable $\mathrm{k}-\varepsilon$ model gives the good approximation near to the wall i.e. $\mathrm{y} / \mathrm{D}=1.06$ to 4 , but small deviation is observed at a distance of $y / D=7$ as indicated in the figure.

To estimate the combined effect of the vortex and boundary layer, in case of near wall for different gap ratio (G/D) similar numerical analysis was performed for Reynolds number 1440 .

It has been observed that, unlike unbounded flow other RANS models are unable to capture the all the secondary vortex formed in the cylinder downstream direction. Hence in the case of near wall flow, k- $\oplus$ SST model is selected with pressure velocity coupling.

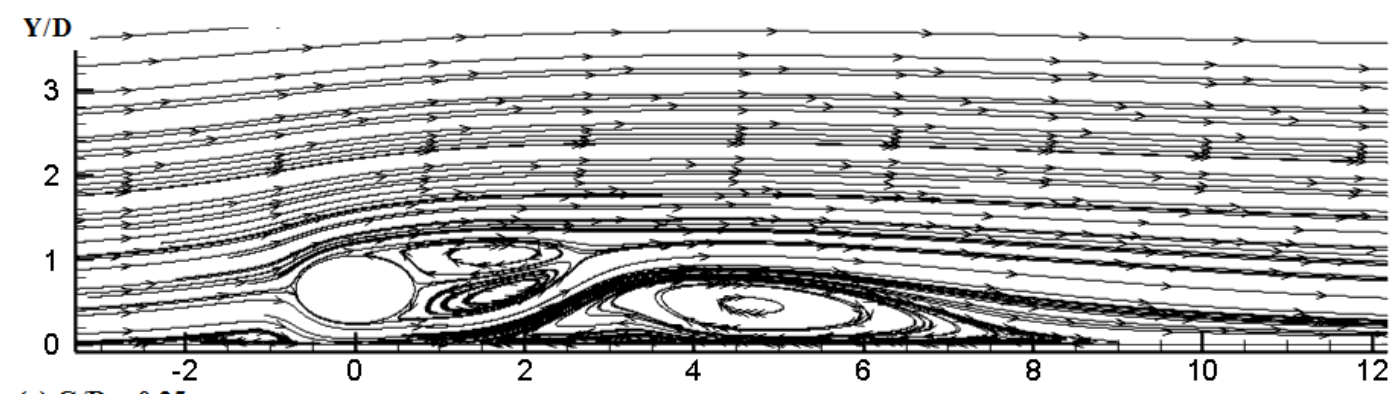

(a) $\mathrm{G} / \mathrm{D}=0.25$

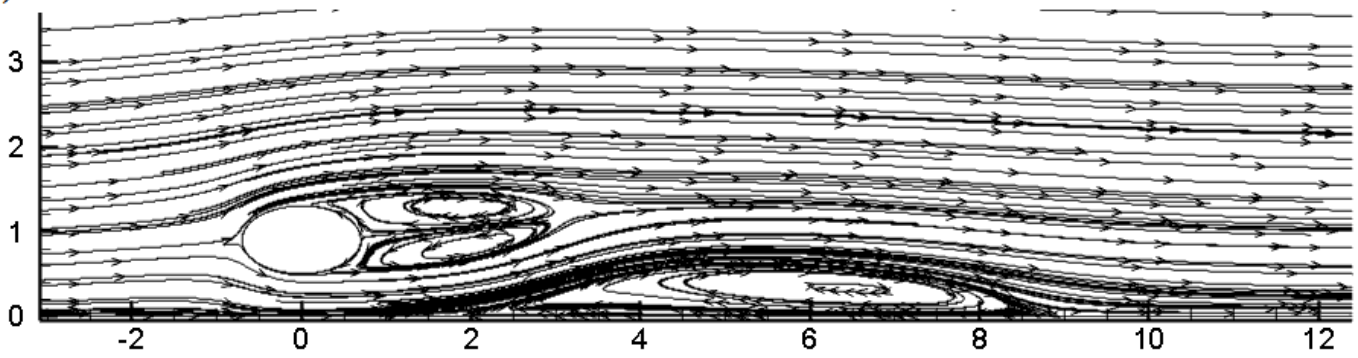

(b) $\mathrm{G} / \mathrm{D}=\mathbf{0 . 5}$

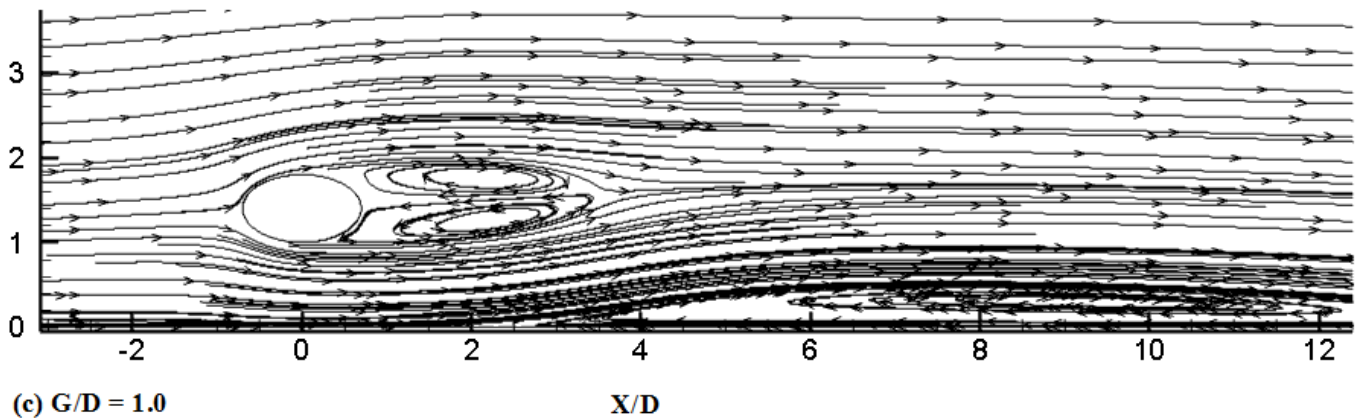

Fig. 5. Mean stream wise velocity contours along with few selected streamlines for different gap ratios 
As indicated in the figure 5, k- $\oplus$ SST turbulence model capture all the primary as well as secondary bubbles in both upstream and downstream direction. As in case of the LES simulation performed by S. Sarkar et al. [3], the upstream bubbles gets reduces in size as the gap ratio increases in case of the present RANS simulation also. Furthermore, it has been observed that the rapid increase in the size of the primary bubble in the downstream direction, as the gap ratio increases. The secondary bubble in the upstream direction gets diminished for highest gap ratio. Primary bubble size increases lengthwise significantly in $\mathrm{X}$ direction by reducing its height in $\mathrm{Y}$ direction. Figure 6, Figure 7 Figure 8, indicates the dynamic pressure profile at different relative gap ratios.

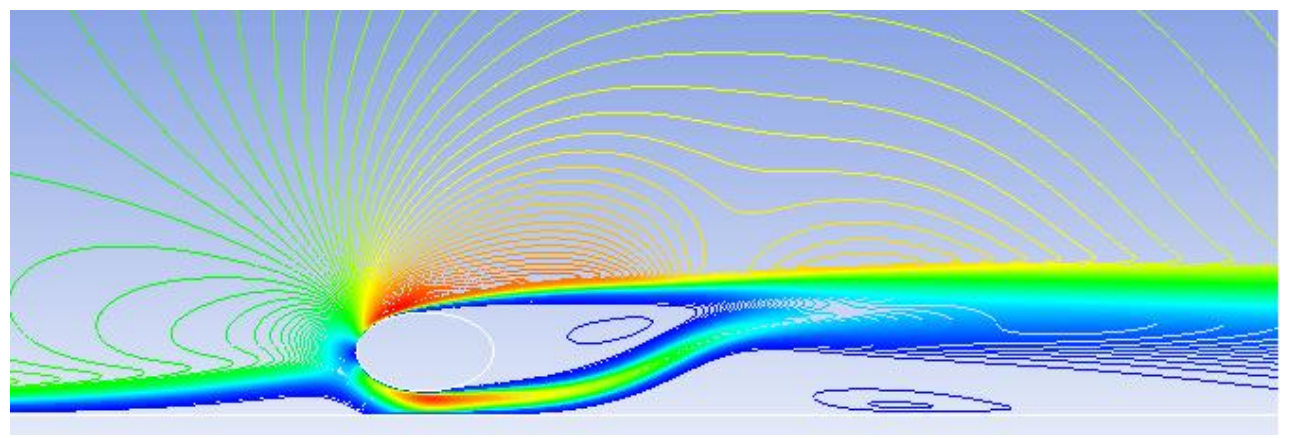

Fig. 6. Dynamic pressure profile at $\mathrm{G} / \mathrm{D}=0.25$

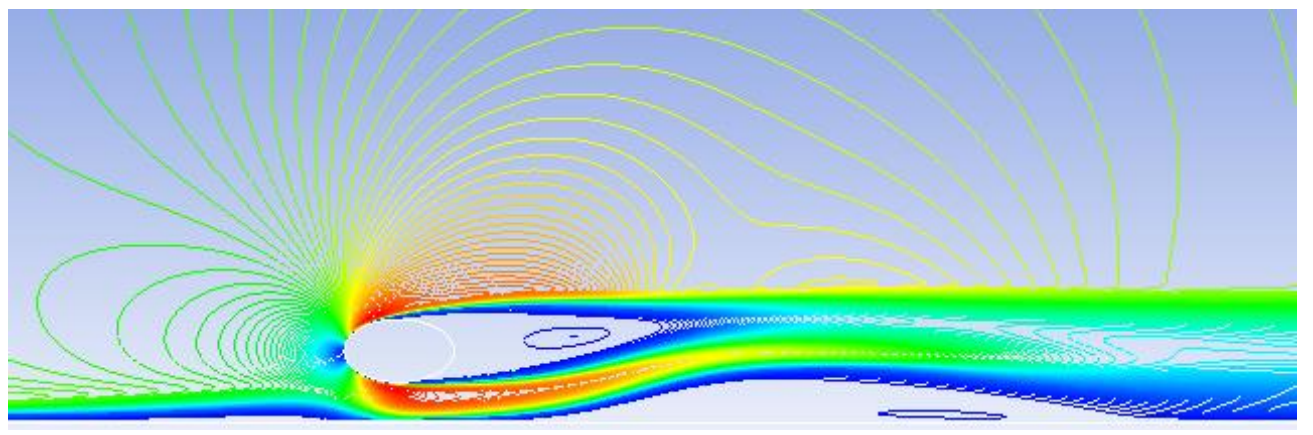

Fig. 7. Dynamic pressure profile at $\mathrm{G} / \mathrm{D}=0.5$

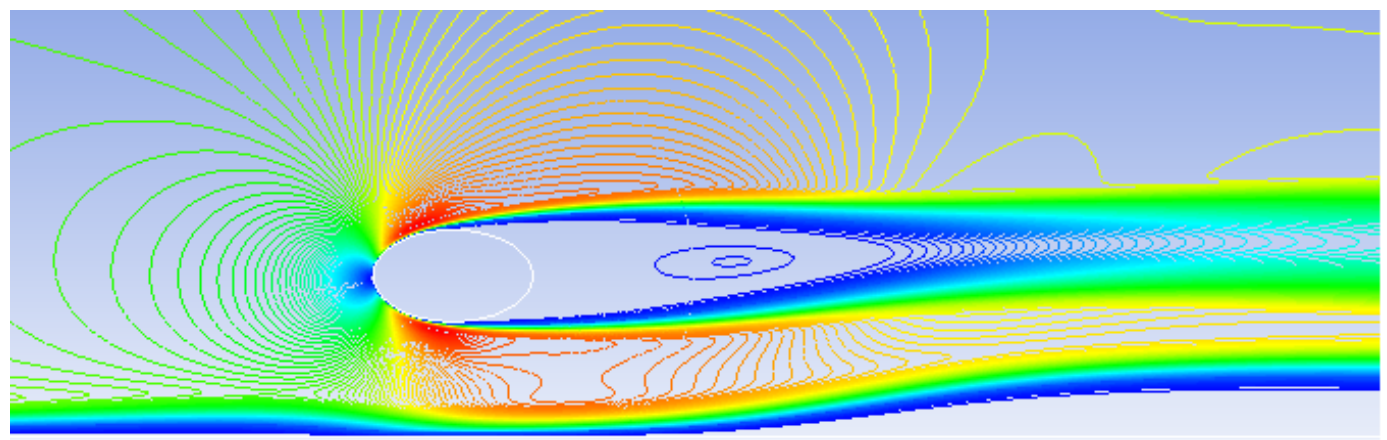

Fig. 8. Dynamic pressure profile at $\mathrm{G} / \mathrm{D}=1.0$ 
A boundary layer may be laminar or turbulent. A laminar boundary layer is one where the flow takes place in layers, i.e., each layer slides past the adjacent layers. Boundary layers appear on the surface of bodies in viscous flow because the fluid seems to attach to the surface. The turbulent kinetic energy is measure of turbulence generated on the air side as the fluid pass over the object.

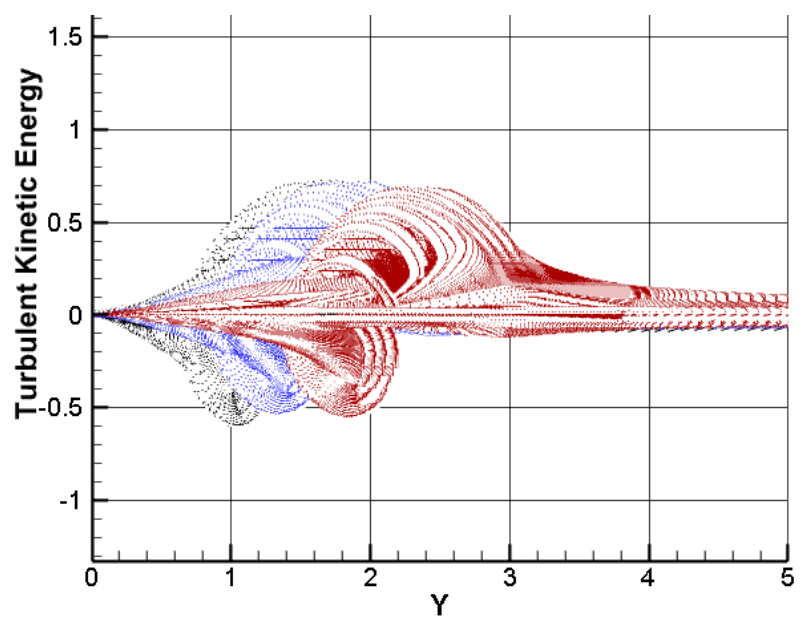

Fig. 9. Turbulance kinetic energy dissipation along the $Y$ axis $(G=0.25 ; G=0.5 ; G=1.0)$

Figure 9 indicates the variation of turbulence kinetic energy along $\mathrm{Y}$ axis. The high variation in the magnitude of the turbulence is often due to the high pressure drop in the air side by increasing the drag at the cylinder downstream. The intensity of the turbulent kinetic energy will further increase if the gap between the domain wall and the side of the square cylinder is reduced. This increased turbulence will accelerate the bubble formation on either side of the object.

\section{Conclusions}

The RANS numerical analysis of flow past cylinder in the unbounded case and in the vicinity of a wall have been carried. In case of the unbounded case $\mathrm{k}-\varepsilon$ Realizable model gives good agreement with that of the experimental findings. With reference to the circular cylinder same analysis is carried out for elliptical object with different relative gap distances by maintaining same hydraulic diameter. In case of the object with near wall for different gap ratios the flow is simulated for Reynolds number of 1440. The domain wall has the least effect in case of gap ratio of 1.0, while the gap "ratio" of 0.25 has the maximum impact on the wake and bubble formation. The primary bubble is observed in the upstream direction in case of gap ratio of 0.25 only.

\section{Nomenclature :}

RANS équations: Reynolds-Averaged Navier-Stokes équations 


\section{Acknowledgements}

We express our sincere thanks to the Department of Mechanical Engineering, Visvesvaraya National Institute of Technology, Nagpur, for providing the platform through Summer school in CFD 2016.

\section{References}

1. M. Rahman, Md. Mashud Karim, Md. Abdul Alim, "Numerical investigation of unsteady flow past a circular cylinder 2-D finite volume method", Journal of Naval Architecture and Marine Engineering, (June 2007), 27-42.

2. B. Sachin Paramane, Atul Sharma, "Numerical investigation of heat and fluid flow across a rotating circular cylinder maintained at constant temperature in 2-D laminar flow regime", International Journal of Heat and Mass Transfer 52 (2009) 3205-3216.

3. S. Sarkar, Sudipto Sarkar, "Vortex dynamics of a cylinder wake in proximity to a wall", Journal of Fluids and Structures 26 (2010) 19-40.

4. A. Ahmed, "On the wake of a circular cylinder with nodal and saddle attachment", Journal of Fluids and Structures 26 (2010) 41-49.

5. Lourenco, L.M., Shih, C., "Characteristics of the plane turbulent near wake of a circular cylinder, a particle image velocimetry study", (2000).

6. G. Gandikota, Sakir Amiroudine, Dipankar Chatterjee, Gautam Biswas, "The Effect of Aiding/Opposing Buoyancy on Two-Dimensional Laminar Flow Across a Circular Cylinder”, Numerical Heat Transfer, Part A: Applications: An International Journal of Computation and Methodology, 58: 385-402, (2010).

7. A.Sharma, V. Eswaran, "Effect of channel-confinement and aiding/opposing buoyancy on the two-dimensional laminar flow and heat transfer across a square cylinder”, International Journal of Heat and Mass Transfer 48 (2005) 5310-5322 